\title{
BMJ Open Incidence and risk factors of depressive symptoms in 4 years of follow-up among mid-aged and elderly community-dwelling Chinese adults: findings from the China Health and Retirement Longitudinal Study
}

\author{
Yue Wen, ${ }^{1}$ Chunjuan Liu, ${ }^{1}$ Jing Liao, ${ }^{1}$ Yiqiong Yin, ${ }^{1}$ Dongmei $\mathrm{Wu}^{\odot 2,3}$
}

To cite: Wen Y, Liu C, Liao J, et al. Incidence and risk factors of depressive symptoms in 4 years of follow-up among mid-aged and elderly community-dwelling Chinese adults: findings from the China Health and Retirement Longitudinal Study. BMJ Open 2019;9:e029529. doi:10.1136/ bmjopen-2019-029529

- Prepublication history for this paper is available online To view these files, please visit the journal online (http://dx.doi org/10.1136/bmjopen-2019029529).

Received 06 February 2019

Revised 18 July 2019

Accepted 19 July 2019

\section{Check for updates}

(C) Author(s) (or their employer(s)) 2019. Re-use permitted under CC BY-NC. No commercial re-use. See rights and permissions. Published by BMJ.

For numbered affiliations see end of article.

\section{Correspondence to}

Professor Yiqiong Yin; yiqiong489@163.com

Dr Dongmei Wu; wudongmei_2001@163.com

\section{ABSTRACT}

Objectives The purpose of this study was to examine the incidence of depressive symptoms, and determine if baseline risk factors conferred a risk for incident depressive symptoms in nationally representative sample of mid-aged and elderly Chinese adults.

Design This study was a secondary analysis of a prospective cohort from a nationally representative sample.

Setting Community samples were recruited from the baseline survey of the China Health and Retirement Longitudinal Study. A four-stage, stratified, cluster probability sampling strategy was used, which included 10257 households with members aged 45 years or older and their spouse.

Participants A total of 11533 participants free of depressive symptoms at baseline were identified, and 10288 were re-examined in either the first and/or the second follow-up surveys. The current analysis was conducted among the 10288 participants.

\section{Primary and secondary outcome}

measures Depressive symptoms were measured by the Center for Epidemiological Studies Depression Scale short form.

Results The findings showed that the incidence of depressive symptoms in a 4-year follow-up was as high as $22.3 \%$. The incidence was much higher in rural areas $(25.7 \%)$ and in women $(27.9 \%)$. Furthermore, participants with 1 hour longer of night-time sleep had a $10 \%$ lower risk of developing depressive symptoms. Compared with individuals who perceived their health status as poor, those who perceived their health status as excellent had a $62 \%$ lower risk of developing depressive symptoms. In addition, having diabetes $(\mathrm{OR}=1.19)$, chronic kidney disease $(O R=1.32)$, chronic digestive disorders $(O R=1.15)$ and arthritis $(\mathrm{OR}=1.43)$ at baseline increased the risk of depressive symptoms. However, baseline body mass index was not associated with the subsequent depressive symptoms in this population.

Conclusions This study highlights the importance of developing an appropriate screening test to identify depressive symptoms for those who are vulnerable and
Strengths and limitations of this study

- Since the sample was representative of community-dwelling mid-aged and older Chinese adults, the study results can be generalised to mid-aged and older Chinese adults.

- Depressive symptoms were based on self-reports instead of clinical diagnosis. Therefore, information bias may be present. However, with a longitudinal design, information bias in the current study was likely to be non-differential.

- This study was a prospective analysis of baseline risk factors of depressive symptoms at follow-up, and no causal associations between these predictors and depressive symptoms can be drawn from the analysis.

ensure these individuals can receive early interventions for depressive symptoms.

\section{INTRODUCTION}

Depression has been recognised as a leading cause of disability and a major contributor to disease burdens globally. ${ }^{1}$ An estimated 322 million adults had depression in 2015 worldwide, with nearly half of these people living in the South-East Asia and Western Pacific regions. ${ }^{2}$ In China, about $4.2 \%$ of adults were estimated to be depressed, and the prevalence of depression reached a peak in older adulthood. ${ }^{2}$ With fast economic growth, mid-aged adults are exposed to a high-stress lifestyle, which is thought to contribute significantly to an increase in mental health disorders in susceptible people. ${ }^{1}$ Older adults, on the other hand, face noteworthy challenges to their life, including loss of independence, loss of social support due to the death of a spouse or weakened family connectedness 
(traditionally referred to as 'filial piety'), financial difficulties and medical vulnerability. ${ }^{3}$ Late-life depressive symptoms increase risk for significant impairment in social function, ${ }^{3}$ dementia, ${ }^{4}$ declined quality of life ${ }^{5}$ and suicide. ${ }^{6}$ They further complicate the prognosis of concurrent medical problems by increasing physical disability and decreasing motivation and adherence to prescribed medications and/or exercise or rehabilitation programmes. ${ }^{78}$

Although depression has come to be regarded as the common cold of psychosocial functioning in Western culture, mental disorders are still viewed as degrading to the patient and to the entire family in Chinese culture. ${ }^{9} 10$ In addition, although effective interventions have been developed to alleviate symptoms of depression, depressive symptoms are often overlooked among the elderly, since most of them mistakenly consider these symptoms to be part of the normal ageing process. ${ }^{11}$ Therefore, older adults may not actively seek medical treatment and leave their depressive symptoms undiagnosed or untreated. As persons aged 45 or older will increase from $32 \%$ to $51 \%$ of the total population from 2010 to 2040 in China, ${ }^{12}$ reliable and up-to-date estimates of the proportion of this population affected by depressive symptoms are a key ingredient of effective health policy, planning and evaluations.

Recently, a number of population-based studies have estimated the prevalence of depressive symptoms among mid-aged and older Chinese adults. ${ }^{311} 1314$ Given that all these studies were cross sectional, the incidence of depressive symptoms among mid-aged and older Chinese adults was not clear. Furthermore, despite a myriad of studies that examined a number of health conditions associated with an increased risk of mid/late-life depressive symptoms, including chronic diseases, overweight/obesity, sleep duration and self-perceived health status, ${ }^{314-17}$ the prospective relationships between these health conditions and depressive symptoms have not been well understood in this population. Such prospective relationships may provide further evidence on how baseline conditions may be predictive of depressive symptoms in the follow-up periods than cross-sectional analysis, especially given that these health conditions are expected to be a great challenge to an increasing ageing population, and can often co-occur with depressive symptoms which may make these conditions even worse. ${ }^{3}$ A better understanding of these associations may also provide information that is useful in identifying patients who are vulnerable to mid-life or latelife depressive symptoms, and proactively assessing and treating modifiable risk factors during primary healthcare service.

Therefore, the purpose of this study was to examine the incidence of depressive symptoms among mid-aged and older adults in China, and determine if baseline sleep duration, body weight, self-perceived health status and chronic conditions conferred a risk for incident depressive symptoms over time, using baseline and 4 years of follow-up data from the China Health and Retirement Longitudinal Study (CHARLS).

\section{METHODS}

\section{Study design}

This study was a secondary analysis of prospective data from the CHARLS. The CHARLS is a nationwide, community-engaged, population-based epidemiological study of Chinese adults aged 45 years or older. The purpose of the CHARLS was to obtain detailed information regarding the dynamics of retirement and how it interacts with health, health insurance and economic well-being. The CHARLS collects detailed information on a wide range of domains, including demographics, health status, physical measures, employment history, pension insurance, retirement, income, expenditures and assets. ${ }^{18}$ The current study used data from baseline, and the first and second follow-up surveys.

\section{Participants}

The CHARLS national baseline survey was conducted in 28 provinces (Tibet, Ningxia and Hainan were not included) across the country from May 2011 to March 2012. It was a survey of 10257 households with members aged 45 years or older and their spouse, for a total of about 17708 individuals. A four-stage, stratified, cluster probability sampling strategy was used to select eligible participants. ${ }^{18}$ Details of the sampling procedures have been published elsewhere. ${ }^{19}$ In brief, in the first stage, all counties were grouped by gross domestic product and by urban or rural regions. In the second stage, counties were stratified and sampled using probabilities proportional to size (PPS). Three rural villages and urban neighbourhoods were randomly selected as primary sampling units (PSU) using PPS in each county. In the third stage, all buildings in each PSU were recognised on satellite imagery of the Google Earth. A sample of 24 households was randomly selected among all households in each PSU. In the final stage, a short screening form was employed to ensure study eligibility in selected households and members of 45 years of age or above were invited to enrol into the study. The response rate for the survey was over $80 \%$ (94\% in rural areas and $69 \%$ in urban areas). ${ }^{18}$

The baseline cohort of the CHARLS participants was followed up every 2 years with the same survey questionnaires and biomedical measures. ${ }^{18}$ The first follow-up survey of the CHARLS was fielded between July 2013 and January 2014. Subjects in this study used the same inclusion and exclusion criteria as the original study. A total of 11533 participants free of depressive symptoms at baseline were identified, of whom 9329 participated in the first follow-up survey in 2013-2014, a total of 9157 were examined in the second follow-up survey in 2015-2016 and 10288 were followed in either the first or the second follow-up survey. The current analysis was conducted among the 10288 participants.

\section{Patient and public involvement}

In the current study, we used deidentified data from the CHARLS with no direct involvement of or interaction 
with participants in the design, recruitment or conduct of the original cohort study.

\section{Variables, definitions and measures Depressive symptoms}

The Center for Epidemiological Studies Depression Scale (CES-D) short form was used to measure depressive symptoms. ${ }^{20}$ The CES-D short form consists of 10 items, and each item is rated on a 4-point Likert scale with answers ranging from 0 (rarely or none of the time) to 3 (most or all of the time) and the total possible summary score of 0-30. The time frame for the CES-D short form refers to the week prior to the interview. Item 5 ('feeling hopeful about the future') and item 8 ('feeling happy') are reversely scored before analysis. Higher scores correspond to higher levels of depressive symptoms, and a score of 12 or higher has been used as the cut-off point for depressive symptoms. ${ }^{21}$

The CES-D short form has been validated among a subsample of 742 CHARLS participants aged 60 years and older, demonstrating adequate psychometric properties. ${ }^{21}$ Two factors were identified by confirmatory factor analysis. Somatic retardation and depressive affect were identified as the first factor, and positive affect was identified as the second factor. The two-factor structure varied across both genders in multigroup analysis $\left(\chi^{2}=144.13\right.$, $\mathrm{p}<0.001)$. $\mathrm{R}^{2}$ coefficient was used to measure the reliability of each item, with some of items differing between men and women. For example, depressive affect and somatic retardation explained $37 \%$ and $64 \%$ of the variance in the depression indicator ('bothered') for men and women, respectively. ${ }^{21}$

\section{Measure of health conditions associated with depressive symptoms}

These factors included chronic conditions, overweight/ obesity, sleep duration and self-perceived health status. Information on chronic conditions was primarily based on self-reports except hypertension and diabetes. Participants were asked if they had been diagnosed with any of the following health conditions: arthritis, dyslipidaemia, cancer (excluding minor skin cancers), liver diseases (except for fatty liver, tumours or cancer), cardiovascular diseases (heart attack, coronary heart disease, angina, congestive heart failure or other heart problems), stroke, kidney disease (except for cancer or tumour), stomach or other gastrointestinal diseases (except for tumour or cancer), memory-related diseases and asthma. Participants who answered 'yes' to these questions were defined as having these conditions.

Hypertension was defined based on the most current guidelines from the American Heart Association, which have lowered the threshold of hypertension cut-off points to $130 / 80 \mathrm{~mm} \mathrm{Hg} .{ }^{22}$ A systolic blood pressure measurement of $130 \mathrm{~mm} \mathrm{Hg}$ and higher, or a diastolic measurement of $80 \mathrm{~mm} \mathrm{Hg}$ and higher, or $130 / 80 \mathrm{~mm} \mathrm{Hg}$ and higher, or taking antihypertensive medications including modern Western medicine, traditional Chinese medicine or using other treatment options for hypertension was considered as having hypertension. ${ }^{22}$ Blood pressure was measured three times (approximately $45 \mathrm{~s}$ apart) for each participant on the left arm in a sitting position using an electronic blood pressure monitor (Omron HEM-7112). ${ }^{23}$

The diagnosis of diabetes was based on the current guidelines from the American Diabetes Association. ${ }^{24}$ A reading of $126 \mathrm{mg} / \mathrm{dL}$ or higher for fasting blood glucose, or a reading of $200 \mathrm{mg} / \mathrm{dL}$ or higher for random blood glucose, or a reading of $6.5 \%$ or higher for glycated haemoglobin, or use of insulin, or taking oral hypoglycaemic medications including traditional Chinese medicine, modern Western medicine or other diabetes treatment was considered as having diabetes. ${ }^{24}$

Weight was measured to the nearest $0.1 \mathrm{~kg}$ using a digital scale (Omron HN-286, Yangzhou, China) on an even, uncarpeted surface, with participants removing heavy outer clothing. Height was measured without shoes to the nearest $0.1 \mathrm{~cm}$ using a stadiometer (Seca 213, Hangzhou, China). Body mass index (BMI, weight in kilograms divided by height in metres squared) was calculated from the participants' weight and height.

Self-perceived health status was measured based on self-reports to the following question, 'Would you rate health as...', with five response options provided: excellent, very good, good, fair and poor.

Night-time sleep duration in hours was collected using a question, 'During the past month, how many hours of actual sleep did you get at night (average hours for one night)? (This may be shorter than the number of hours you spend in bed.)'

\section{Covariates}

Factors that could confound the main association were identified a priori from current literature. Covariates included age, gender, ${ }^{3}$ education ${ }^{25}$ and marital status. ${ }^{3}{ }^{36}$ Consistent with a prior CHARLS publication, ${ }^{27} 28$ education was categorised as illiterate or no formal education, some primary school but can read and write, primary school including home schooling and middle school or above. Marital status was grouped as married versus not married.

\section{Statistical analysis}

Incidence of depressive symptoms

The incidence of depressive symptoms was analysed taking into account the complex survey design and non-response rate in both estimates and the corresponding SEs. Participants were categorised into 5 years of age group. SAS PROC SURVEYFREQ procedure was used to obtain overall and gender-specific incidence of depressive symptoms among all participants and by the 5 years of age group. In addition, overall and gender-specific incidence of depressive symptoms was estimated by rural and urban areas. We estimated the incidence of four outcomes of depressive symptoms: having depressive symptoms in the first follow-up survey (2013-2014); having depressive symptoms in the second follow-up 
survey (2015-2016); ever having depressive symptoms in the first or the second follow-up survey; and consistently having depressive symptoms in both follow-up surveys.

\section{Prospective analysis}

Baseline characteristics of the participants were summarised as frequency and percentage for categorical variables and mean and SD or median and IQR for continuous variables. Associations of baseline health conditions, including chronic conditions, overweight/ obesity, sleep duration and self-perceived health status, with the incidence of ever having depressive symptoms in 4 years of follow-up were evaluated by a multivariate logistic regression model, while controlling for all covariates as mentioned above. ORs and the corresponding 95\% CIs were reported. SAS V.9.4 (SAS Institute) was used to perform the analyses. All the $p$ values were two sided, and $\mathrm{p}<0.05$ was considered significant.

\section{RESULTS}

\section{Sample characteristics}

A total of 10288 participants aged 45 years or older were included in the analysis. As shown in table 1, the average age of all participants was 58.5 years and roughly half participants $(49.3 \%)$ were women. Most of participants $(73.3 \%)$ lived in rural areas and $60.6 \%$ of participants had primary education or above. The majority of participants $(90.0 \%)$ were married or living with a partner at the time of data collection. About a third of the participants $(31.5 \%)$ were current smokers and $17.9 \%$ were current regular drinkers. Participants had an average BMI of $23.7 \mathrm{~kg} / \mathrm{m}^{2}$. The mean duration of sleep per night was 6.7 hours, and less than $20 \%$ of participants perceived their health status as very good or excellent. The mean CES-D short form score was 5.1 at baseline.

\section{Incidence of depressive symptoms}

As shown in table 2 and figure 1, the incidence of ever having depressive symptoms in 4 years was $22.3 \%$ (95\% CI $21.3 \%$ to $23.3 \%$ ). The incidence was much higher in rural areas than that in urban areas $(25.7 \%$ vs $15.3 \%$, $\mathrm{p}<0.0001)$. Women had a higher incidence of depressive symptoms than men $(27.9 \%$ vs $16.7 \%, \mathrm{p}<0.0001)$. There is no clear pattern of the incidence of depressive symptoms over age groups (figure 1). The incidence was highest among the 65-70 years of age group and lowest among the $45-50$ years of age group.

\section{Baseline risk factors and incident depressive symptoms}

As shown in table 3 , in the fully adjusted multivariable logistic regression model, night-time sleep duration and self-perceived health status were both inversely associated with the risk of depressive symptoms. Specifically, participants with 1 hour longer of night-time sleep had a $10 \%$ (95\% CI $7 \%$ to $13 \%, \mathrm{p}<0.0001)$ lower risk of developing depressive symptoms in 4 years of follow-up. Compared with individuals who perceived their health status as poor,
Table 1 Baseline characteristics of the CHARLS participants free of depressive symptoms

\begin{tabular}{|c|c|}
\hline Variable & Distribution \\
\hline$n$ & 10288 \\
\hline Age (years), mean (SD) & $58.5(9.8)$ \\
\hline Male, n (\%) & $5845(50.7)$ \\
\hline Living in rural areas, $\mathrm{n}(\%)$ & 8440 (73.3) \\
\hline \multicolumn{2}{|l|}{ Education, n (\%) } \\
\hline No formal education or illiterate & $2736(23.7)$ \\
\hline Some primary school & $1804(15.7)$ \\
\hline Finished primary school & $2590(22.5)$ \\
\hline Junior high school or above & $4394(38.1)$ \\
\hline Currently married, n (\%) & $10375(90.0)$ \\
\hline \multicolumn{2}{|l|}{ Smoking status, n (\%) } \\
\hline Current smoker & 3629 (31.5) \\
\hline Former smoker & $1034(9.0)$ \\
\hline Never smoking & $6868(60.0)$ \\
\hline \multicolumn{2}{|l|}{ Drinking status, $\mathrm{n}(\%)$} \\
\hline Regular drinkers & 1951 (17.9) \\
\hline Occasional drinkers & $476(4.4)$ \\
\hline Former drinkers & $646(5.9)$ \\
\hline Never drinking & $7833(71.8)$ \\
\hline Weight (kg), mean (SD) & $60(11.8)$ \\
\hline Height $(m)$, mean (SD) & $1.6(0.1)$ \\
\hline Body mass index $\left(\mathrm{kg} / \mathrm{m}^{2}\right)$, mean $(\mathrm{SD})$ & $23.7(4)$ \\
\hline Sleeping duration (hours), mean (SD) & $6.7(1.7)$ \\
\hline CES-D short form score & $5.1(3.3)$ \\
\hline \multicolumn{2}{|l|}{ Self-perceived health status, $n(\%)$} \\
\hline Excellent & $483(4.2)$ \\
\hline Very good & $1744(15.1)$ \\
\hline Good & $4136(35.9)$ \\
\hline Fair & 3977 (34.5) \\
\hline Poor & $1187(10.3)$ \\
\hline \multicolumn{2}{|l|}{ Chronic conditions, n (\%) } \\
\hline Hypertension & $4045(40.3)$ \\
\hline Dyslipidaemia & $1016(9.0)$ \\
\hline Diabetes & $1210(15.0)$ \\
\hline Cancer & $97(0.8)$ \\
\hline Liver disease & $387(3.4)$ \\
\hline Heart disease & $1162(10.1)$ \\
\hline Stroke & $188(1.6)$ \\
\hline Kidney disease & $563(4.9)$ \\
\hline Gastrointestinal disease & $2180(18.9)$ \\
\hline Dementia & $105(0.9)$ \\
\hline Arthritis & 3221 (28.0) \\
\hline Asthma & $312(2.7)$ \\
\hline
\end{tabular}

CES-D, Center for Epidemiological Studies Depression Scale short form; CHARLS, China Health and Retirement Longitudinal Study. 
Table 2 Incidence of depressive symptoms among the CHARLS participants

\begin{tabular}{lllll}
\hline & $\begin{array}{l}\text { Ever having depressive } \\
\text { symptoms }\end{array}$ & $\begin{array}{l}\text { Depressive symptoms in } \\
\text { both visits 2 and 3 }\end{array}$ & $\begin{array}{l}\text { Depressive symptoms in } \\
\text { 2 years }\end{array}$ & $\begin{array}{l}\text { Depressive symptoms in } \\
\text { 4 years }\end{array}$ \\
\hline Overall & $22.3(21.3-23.3)$ & $4.4(4.0-4.9)$ & $13.2(12.5-14.1)$ & $16.8(15.9-17.7)$ \\
Rural & $25.7(24.6-26.8)$ & $5.3(4.8-5.8)$ & $15.3(14.3-16.2)$ & $18.8(17.8-19.8)$ \\
Urban & $15.3(13.4-17.2)$ & $2.6(1.9-3.3)$ & $9.0(7.4-10.6)$ & $12.2(10.4-14.1)$ \\
Men & $16.7(15.5-17.9)$ & $3.0(2.5-3.5)$ & $9.9(9.0-10.9)$ & $12.2(11.1-13.2)$ \\
Women & $27.9(26.4-29.4)$ & $5.8(5.1-6.6)$ & $16.6(15.3-17.9)$ & $21.5(20.1-22.9)$ \\
\hline
\end{tabular}

CHARLS, China Health and Retirement Longitudinal Study.

those who perceived their health status as fair, good, very good and excellent had a $42 \%(95 \%$ CI $39 \%$ to $52 \%)$, $47 \%$ (95\% CI $36 \%$ to $57 \%$ ), $64 \%$ (95\% CI $54 \%$ to $72 \%$ ) and $62 \%$ (95\% CI $43 \%$ to $74 \%$ ) lower risk of developing depressive symptoms, respectively. In addition, four chronic conditions, including diabetes $(\mathrm{OR}=1.19,95 \% \mathrm{CI}$ 1.00 to $1.42, \mathrm{p}=0.04)$, chronic kidney disease $(\mathrm{OR}=1.32$, 95\% CI 1.04 to $1.67, \mathrm{p}=0.02$ ), chronic digestive disorders $(\mathrm{OR}=1.15,95 \% \mathrm{CI} 1.01$ to $1.31, \mathrm{p}=0.04)$ and arthritis $(\mathrm{OR}=1.43,95 \%$ CI 1.28 to $1.61, \mathrm{p}<0.0001)$, were associated with higher risk of depressive symptoms. However, baseline BMI was not associated with the onset of depressive symptoms in this population $(\mathrm{OR}=0.99,95 \%$ CI 0.97 to 1.01$)$. Chronic lung disease $(\mathrm{p}=0.001)$, heart disease $(\mathrm{p}=0.004)$ and asthma $(\mathrm{p}=0.02)$ were positively associated with incidence of depressive symptoms in the raw analysis, but became non-significant in the fully adjusted model (all $\mathrm{p}>0.05$ ).

\section{DISCUSSION}

Through the analysis of data from a nationally representative sample of mid-aged and older Chinese adults, we found that the incidence of depressive symptoms in a 4-year follow-up was as high as $22.3 \%$. Large disparities in the incidence of depressive symptoms were observed between rural and urban areas and between men and women. Furthermore, longer night-time sleep duration

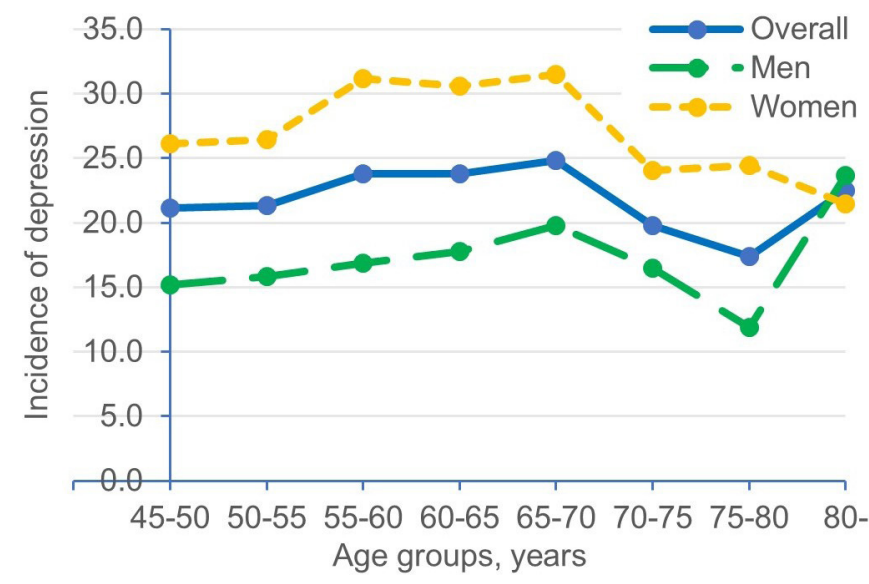

Figure 1 The incidence of depressive symptoms in 4 years of follow-up among participants of the China Health and Retirement Longitudinal Study (CHARLS). and better self-perceived health status were both associated with lower risk of depressive symptoms. In addition, diabetes, chronic kidney disease, chronic digestive disorders and arthritis at baseline increased the risk of depressive symptoms. However, baseline BMI was not associated with depressive symptoms in this population.

Our study found that in 4 years of follow-up, about $22.3 \%$ of the mid-aged and older Chinese adults developed depressive symptoms. Using the same database, a recent study reported that the prevalence of depressive symptoms at CHARLS baseline survey was up to 26.2\%. ${ }^{13}$ Collectively, these findings revealed that depressive symptoms were a major public health challenge in China, and intervention programmes should be developed to reduce the burden of depressive symptoms. Furthermore, large disparities were also observed between rural and urban residents and between men and women. In particular, women and residents living in rural areas were at a higher risk of developing depressive symptoms. The high incidence rate was relevant to mid-aged and older Chinese adults, and had implications for young children's developmental outcomes. Many depressive symptom cases remained undiagnosed, and even among those who were aware of the condition, few of them sought treatment due to cultural stigma. ${ }^{9}$ Mid-aged and elderly grandparents are the primary caregivers of infants, toddlers and young children in rural China. Depressed caregivers were more likely to show negative parenting interactions (eg, limited facial and behavioural affect) with their children, and these interactions were directly relevant for children's development. ${ }^{29}{ }^{30}$ A strong negative correlation has been reported between the levels of depression of grandmothers and the levels of developmental outcomes of the grandchildren in their care, including cognitive, language, social-emotional and motor functions. $^{31} 32$ Taken together, changes in social policies and intervention programmes should be developed to address timely diagnosis and treatment of depression for these people, which will help improve young children's developmental outcomes in rural China.

Individuals with longer night-time sleep duration had a lower risk of depressive symptoms. This finding is in line with previous studies. ${ }^{33} 34$ Sleep disorders are often the presenting and core symptoms of depression. ${ }^{35}$ However, 
Open access

Table 3 Raw and multivariate adjusted associations with the incidence of depressive symptoms in 4 years of follow-up

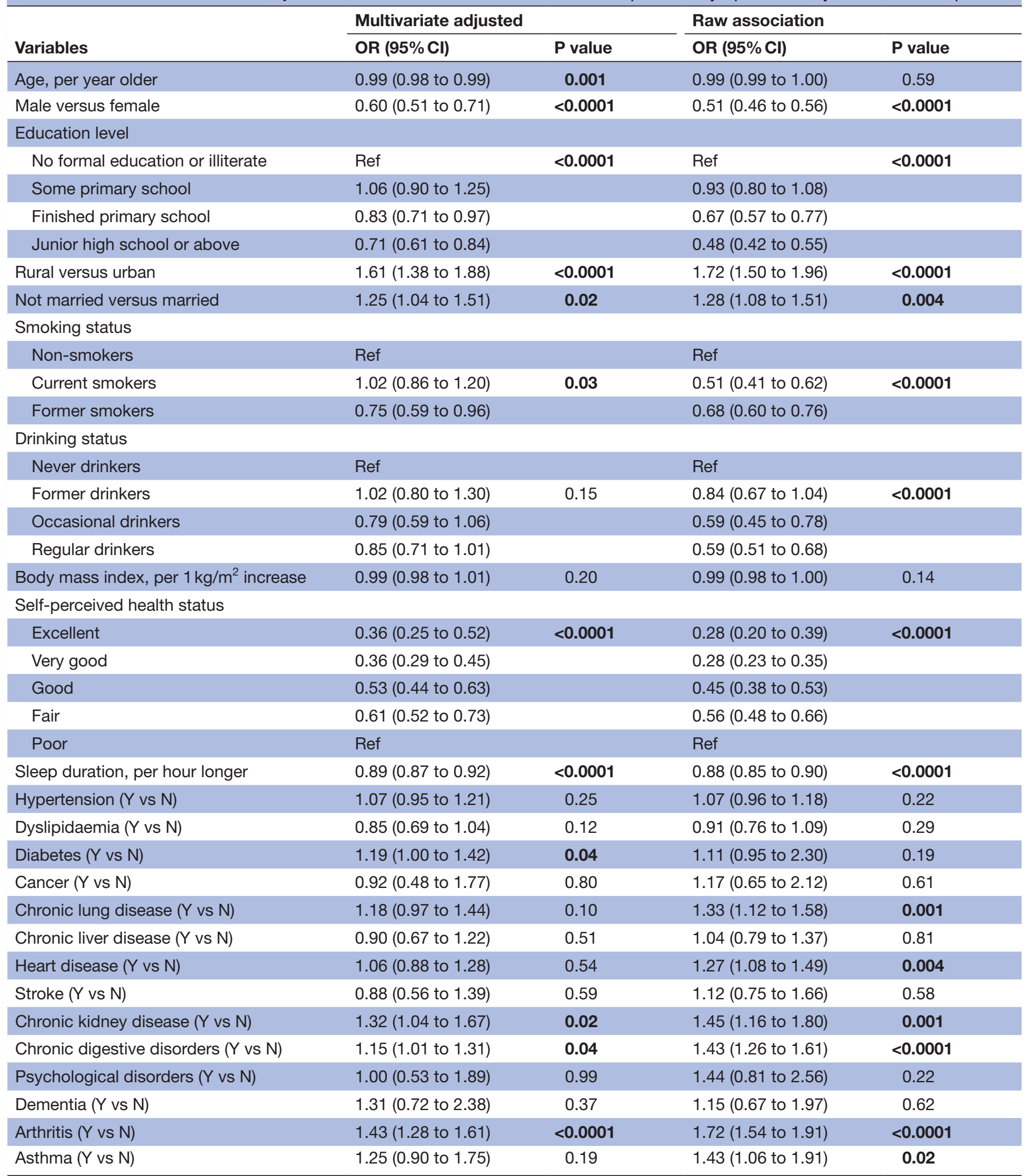

All analyses were conducted at a $5 \%$ significance level.

Bold values denote significant findings.

treatment of depression did not resolve sleep symptoms, which may confer a greater risk for depression recurrence and relapse. ${ }^{35}$ Therefore, sleep deprivation should be at least considered as an early indicator of depressive symptoms. Individuals with sleep deprivation should be aware 
of their high risk of depressive symptoms and increase night-time sleep duration.

Better self-perceived health status at baseline was associated with a lower risk of depressive symptoms in the current study. The finding is consistent with studies among other populations. ${ }^{36} 37$ Self-perceived health is a powerful indicator of the overall health status of elderly people. ${ }^{38}$ Many factors, including sociodemographic characteristics, chronic diseases, functional status, social relationships, neighbourhood environment and nutrition pattern, are all important determinants of self-perceived health status. ${ }^{38}$ These factors are also associated with depressive symptoms. The current study adjusted most of the factors, except for functional status, social relationship and nutrition pattern, which were not available in the CHARLS database. However, poorer self-perceived health status was still significantly associated with a higher risk of depressive symptoms. Future studies additionally controlling for those factors will help evaluate the contribution of those factors to the association of self-perceived health status with depressive symptoms. Furthermore, such study may also help delineate whether self-perceived health status may represent other factors that may lead to depressive symptoms. Finally, previous studies also found that individuals with depressive disorders were more likely to report poor self-perceived health status. ${ }^{39} 40$ Therefore, there might be a bidirectional association between depressive symptoms and self-perceived health status. Future studies examining the bidirectional relationship are therefore needed.

Among 12 chronic conditions, baseline chronic kidney disease increased the risk of depressive symptoms at follow-up. Although the underlying mechanisms have not been well understood, some authors suggested that the psychosocial and biological changes in dialysis may explain this relationship. ${ }^{41}$ In addition to chronic kidney disease, baseline diabetes also conferred a higher risk of symptoms of depression over time in this study. Similar results were reported in a meta-analysis of 11 studies, which found that people with diabetes at baseline had a $24 \%$ higher risk of developing depression at follow-up compared with those without diabetes. ${ }^{42}$ Furthermore, a high prevalence of depression among people with diabetes and/or chronic kidney disease has also been reported in cross-sectional studies. ${ }^{43-45}$ In concordance with these findings, this study added further evidence that compared with other chronic conditions at baseline, baseline chronic kidney disease and diabetes were more likely to be associated with depressive symptoms over time in a population of mid-aged and older Chinese adults.

Consistent with our study, a growing body of evidence reveals a high prevalence and incidence of depression among people with arthritis. For example, in a recent meta-analysis, the prevalence of major depressive disorder among patients with rheumatoid arthritis was $17 \%{ }^{46}$ In a population-based cohort in Taiwan, patients with rheumatoid arthritis were 2.06 times more likely to develop depression compared with the control patients. ${ }^{47}$ The high prevalence and incidence of depression among patients with arthritis may be attributed to several factors, including the impact of the diagnosis with no cure, arthritis symptoms and flare-ups, loss of work productivity and side effects of medications. ${ }^{48}$ In the current study, we found that chronic digestive disorders at baseline conferred a higher risk for depressive symptoms over time. In line with this finding, using the National Health Insurance Research Database in Taiwan, Lee et al found that irritable bowel syndrome was a risk factor for subsequent depressive and anxiety disorders. $^{49}$ The digestive system is sensitive to the impact of emotional factors, because the function of digestive system is jointly controlled by the endocrine system and vegetative nervous system, and the centre of both systems is located at the same place as the centre of emotion. ${ }^{50}$

Overweight/obesity is well established to interfere with mental health, with depressive symptoms being more common among overweight/obese individuals than their normal-weight counterparts, especially in overweight/obese women. ${ }^{51} 52$ This relationship was further confirmed by a meta-analysis of longitudinal studies, in which excess weight at baseline increased a higher risk for subsequent depression at follow-up. ${ }^{53}$ In contrast to these findings from Western and European countries, using data from a prospective survey of a large representative sample of mid-aged and elderly community-dwelling residents in mainland China, we did not find a relationship between baseline BMI and subsequent depressive symptoms over time. These inconsistent findings may suggest that because participants in the current study were predominantly low in BMI at baseline (mean baseline BMI was $23.7 \mathrm{~kg} / \mathrm{m}^{2}$ ), their likelihood of experiencing depressive symptoms may have been lower compared with those participants reported in Luppino et al $\mathrm{s}^{53}$ study. However, these are preliminary findings that need to be further investigated in future prospective studies in this population.

The study has several important strengths. First, to the best of our knowledge, this is the first study that examined the incidence of depressive symptoms in a nationally representative population in China. As noted above, previous studies have primarily focused on the prevalence of depressive symptoms ${ }^{311} 1314$ or investigated the incidence of depressive symptoms in a regional sample. ${ }^{54}$ Second, since the sample was representative of community-dwelling mid-aged and older Chinese adults, the results can be generalised to mid-aged and older Chinese adults. Third, a Chinese specific cut-off point of the CES-D short form has been used, and the cut-off point was validated among mid-aged and older Chinese adults. ${ }^{21}$ Therefore, our study provided a more reliable estimate of the incidence of depressive symptoms. Finally, strict quality control measures, including global positioning system matching, data checking, 
recording and checking interviews, and calling back participants, were implemented at each stage of the CHARLS to ensure high-quality data.

There are also some limitations in this study that should be acknowledged. First, depressive symptoms were based on self-reports instead of clinical diagnosis. Therefore, information bias may be present. However, with a longitudinal design, information bias in the current study was likely to be non-differential. Since non-differential misclassification generally affects the association estimates towards the null, our study findings were more robust. Second, night-time sleep duration was measured based on self-reports. The use of self-report measures in the CHARLS surveys may have biased study results, since participants may consistently underestimate or overestimate their night-time sleep duration. ${ }^{55}$ Objective or direct measures of sleep duration should be used to increase precision and accuracy of self-report assessment methods. Finally, this study was a prospective analysis of baseline risk factors of depressive symptoms at follow-up, and no causal associations between these predictors and depressive symptoms can be drawn from the analysis.

The findings of the current study have important implications for clinical practice. Healthcare professionals are encouraged to address the needs for better mental health in older adults. ${ }^{56}$ In view of the high incidence of depressive symptoms (22.3\%) among mid-aged and elderly Chinese adults, the findings of this study highlight the importance of developing an appropriate screening test to identify depressive symptoms for those who are vulnerable and ensure these individuals can receive early interventions for depressive symptoms. The screening test should include items such as areas of living (rural or urban areas), gender, night-time sleep duration, self-perceived health status and certain chronic conditions, especially chronic kidney disease, diabetes, arthritis and chronic digestive disorders. In addition, intervention strategies that address short night-time sleep duration may be used to alleviate depressive symptoms and improve mental health in this population. Intervention programmes should also be prioritised to target at specific populations, such as women and residents living in rural areas, as these populations were at a higher risk of developing depressive symptoms. Finally, new patients with chronic kidney disease, diabetes, arthritis or chronic digestive disorders should be screened for depressive symptoms using the CES-D short form at the initial visit. Persons with depressive symptoms (a score of 12 or higher) should then be assessed by a clinician using the structured clinical interview for Diagnostic and Statistical Manual of Mental Disorders, Fifth Edition. Potential participants with serious depressive symptoms should be referred immediately to a mental health professional. Individuals with these chronic conditions are particularly vulnerable to the deleterious effects of affective symptoms, since symptoms of depression are a known risk factor for non-compliance with medical treatment and adverse health outcomes. ${ }^{48} 495758$

\section{CONCLUSIONS}

To conclude, our study found a high incidence of depressive symptoms in 4 years of follow-up among a nationally representative mid-aged and older Chinese population. In addition, great disparities in the incidence of depressive symptoms were observed between individuals living in rural and urban areas and between men and women. Furthermore, we identified that worse self-perceived health, shorter night-time sleep duration, diabetes, chronic kidney disease, chronic digestive disorders and arthritis at baseline increased the risk for depressive symptoms. These findings supported the importance of baseline status to longitudinal changes in depressive symptoms. Interventions for vulnerable mid-aged and older adults should focus on addressing short night-time sleep duration. These findings also help identify individuals at a higher risk for depressive symptoms, such as those with chronic kidney disease, chronic digestive disorders, arthritis and diabetes, or women and residents living in rural areas, so that early interventions can be implemented to prevent depressive symptoms in these people.

\section{Author affiliations}

${ }^{1}$ Department of Gastrointestinal Surgery, West China Hospital, Sichuan University, Chengdu, China

${ }^{2}$ The Clinical Hospital of Chengdu Brain Science Institute, MOE Key Lab for Neuroinformation, University of Electronic Science and Technology of China, Chengdu, China

${ }^{3}$ Psychiatric Research Laboratory, The Fourth People's Hospital of Chengdu and Chengdu Mental Health Center, Chengdu, China

Acknowledgements The authors gratefully acknowledge the assistance of $\mathrm{Dr}$ Tingting Liu, an assistant professor at University of Arkansas Eleanor Mann School of Nursing, for editing the manuscript.

Contributors All authors contributed to the conception and design of this study. YW, YY and DW were responsible for the design, analysis, drafting and revision of this manuscript. CL and $\mathrm{JL}$ were responsible for interpretation of data and preparation of the manuscript.

Funding This work was supported by the Sichuan Science and Technology Program (grant number 2018JY0306 to DW). The CHARLS is funded by Peking University, the National Natural Science Foundation of China, the Behavioral and Social Research Division of the National Institute on Aging, and the World Bank.

\section{Competing interests None declared.}

\section{Patient consent for publication Not required}

Ethics approval The CHARLS was approved by the Peking University Ethical Review Committee. The current study is a secondary analysis of the deidentified CHARLS public data. The Ethics Review Committee granted an exempt research determination to the current study.

Provenance and peer review Not commissioned; externally peer reviewed.

Data availability statement Data are available in a public, open access repository.

Open access This is an open access article distributed in accordance with the Creative Commons Attribution Non Commercial (CC BY-NC 4.0) license, which permits others to distribute, remix, adapt, build upon this work non-commercially, and license their derivative works on different terms, provided the original work is properly cited, appropriate credit is given, any changes made indicated, and the use is non-commercial. See: http://creativecommons.org/licenses/by-nc/4.0/. 


\section{REFERENCES}

1. Qin X, Wang S, Hsieh C. The prevalence of depression and depressive symptoms among adults in China: estimation based on a national household survey. China Economic Review 2016.

2. World Health Organization. Depression and other common mental disorders: global health estimates. Geneva, Swiss: World Health Organization, 2017.

3. Lei X, Sun X, Strauss J, et al. Depressive symptoms and Ses among the mid-aged and elderly in China: evidence from the China health and retirement longitudinal study national baseline. Soc Sci Med 2014:120:224-32.

4. Diniz BS, Butters MA, Albert SM, et al. Late-Life depression and risk of vascular dementia and Alzheimer's disease: systematic review and meta-analysis of community-based cohort studies. Br J Psychiatry 2013;202:329-35.

5. Sivertsen H, Bjørkløf GH, Engedal K, et al. Depression and quality of life in older persons: a review. Dement Geriatr Cogn Disord 2015;40:311-39.

6. Wang C-W, Chan CLW, Yip PSF. Suicide rates in China from 2002 to 2011: an update. Soc Psychiatry Psychiatr Epidemiol 2014:49:929-41.

7. Holzapfel N, Löwe B, Wild B, et al. Self-Care and depression in patients with chronic heart failure. Heart Lung 2009;38:392-7.

8. Gonzalez JS, Safren SA, Delahanty LM, et al. Symptoms of depression prospectively predict poorer self-care in patients with type 2 diabetes. Diabet Med 2008;25:1102-7.

9. Zeng B, Sun W, Gary RA, et al. Towards a conceptual model of diabetes self-management among Chinese immigrants in the United States. Int J Environ Res Public Health 2014;11:6727-42.

10. He P, Hu Y, Li C, et al. Predictors of depressive symptoms among mid-aged and older men with diabetes in China. Res Theory Nurs Pract 2019;33:6-22.

11. Xu Y, Yang J, Gao J, et al. Decomposing socioeconomic inequalities in depressive symptoms among the elderly in China. BMC Public Health 2016;16:1214.

12. United Nations Deparment of Economic and Social Affais/Population Division. World population prospects: the 2012 revision, volume II: Demophic profiles. New York, NY: United Nations, 2012.

13. Li C, Miles T, Shen L, et al. Early-Life exposure to severe famine and subsequent risk of depressive symptoms in late adulthood: the China health and retirement longitudinal study. Br J Psychiatry 2018;213:579-86.

14. Yu J, Li J, Cuijpers P, et al. Prevalence and correlates of depressive symptoms in Chinese older adults: a population-based study. Int $J$ Geriatr Psychiatry 2012;27:305-12.

15. Alvaro PK, Roberts RM, Harris JK. A systematic review assessing bidirectionality between sleep disturbances, anxiety, and depression. Sleep 2013;36:1059-68.

16. Wilson-Genderson M, Heid AR, Pruchno R. Onset of multiple chronic conditions and depressive symptoms: a life events perspective. Innov Aging 2017;1.

17. Qian J, Li N, Ren X. Obesity and depressive symptoms among Chinese people aged 45 and over. Sci Rep 2017;7:45637.

18. Zhao Y, Hu Y, Smith JP, et al. Cohort profile: the China health and retirement longitudinal study (CHARLS). Int $J$ Epidemiol 2014;43:61-8.

19. Li C, Liu T, Sun W, et al. Prevalence and risk factors of arthritis in a middle-aged and older Chinese population: the China health and retirement longitudinal study. Rheumatology 2015;54:697-706.

20. Andresen EM, Malmgren JA, Carter WB, et al. Screening for depression in well older adults: evaluation of a short form of the CES-D (center for epidemiologic studies depression scale). Am J Prev Med 1994;10:77-84.

21. Chen $\mathrm{H}$, Mui AC. Factorial validity of the center for epidemiologic studies depression scale short form in older population in China. Int Psychogeriatr 2014;26:49-57.

22. Whelton PK, Carey RM, Aronow WS, et al. 2017 ACC/AHA/AAPA/ ABC/ACPM/AGS/APhA/ASH/ASPC/NMA/PCNA guideline for the prevention, detection, evaluation, and management of high blood pressure in adults: Executive summary: a report of the American College of Cardiology/American heart association Task force on clinical practice guidelines. Circulation 2018;138:e426-83.

23. Zhao Y, Crimmins $\mathrm{E}, \mathrm{Hu} \mathrm{P}$, et al. China health and retirement longitudinal study: 2011-2012 national baseline blood data users' guide. Beijing, China: China Center for Economic Research, Peking Universtiy, 2014

24. American Diabetes Association. 2. Classification and Diagnosis of Diabetes: Standards of Medical Care in Diabetes-2018. Diabetes Care 2018;41(Suppl 1):S13-27.
25. Bjelland I, Krokstad S, Mykletun A, et al. Does a higher educational level protect against anxiety and depression? the HUNT study. Soc Sci Med 2008;66:1334-45.

26. Cheng HG, Chen S, McBride O, et al. Prospective relationship of depressive symptoms, drinking, and tobacco smoking among middle-aged and elderly community-dwelling adults: results from the China health and retirement longitudinal study (CHARLS). J Affect Disord 2016;195:136-43.

27. Zuo M, Gan C, Liu T, et al. Physical predictors of cognitive function in individuals with hypertension: evidence from the CHARLS basline survey. West J Nurs Res 2019;41:592-614.

28. Zhang M, Liu T, Li C, et al. Physical performance and cognitive functioning among individuals with diabetes: findings from the China health and retirement longitudinal study baseline survey. J Adv Nurs 2019;75:1029-41.

29. Lovejoy MC, Graczyk PA, O'Hare E, et al. Maternal depression and parenting behavior: a meta-analytic review. Clin Psychol Rev 2000;20:561-92.

30. Pelaez M, Field T, Pickens JN, et al. Disengaged and authoritarian parenting behavior of depressed mothers with their toddlers. Infant Behav Dev 2008;31:145-8.

31. Yue A, Gao J, Yang M, et al. Caregiver depression and early child development: a mixed-methods study from rural China. Front Psychol 2018;9:2500.

32. Zhang S, Dang R, Yang N, et al. Effect of caregiver's mental health on early childhood development across different rural communities in China. Int J Environ Res Public Health 2018;15:2341.

33. Sun Y, Shi L, Bao Y, et al. The bidirectional relationship between sleep duration and depression in community-dwelling middle-aged and elderly individuals: evidence from a longitudinal study. Sleep Med 2018;52:221-9.

34. Li Y, Wu Y, Zhai L, et al. Longitudinal association of sleep duration with depressive symptoms among middle-aged and older Chinese. Sci Rep 2017;7:11794.

35. Nutt D, Wilson S, Paterson L. Sleep disorders as core symptoms of depression. Dialogues Clin Neurosci 2008;10:329-36.

36. Cole MG, Dendukuri N. Risk factors for depression among elderly community subjects: a systematic review and meta-analysis. $A m$ Psychiatry 2003;160:1147-56.

37. Conti CL, Barbosa WM, Simão JBP, et al. Pesticide exposure, tobacco use, poor self-perceived health and presence of chronic disease are determinants of depressive symptoms among coffee growers from Southeast Brazil. Psychiatry Res 2018;260:187-92.

38. Machón M, Vergara I, Dorronsoro M, et al. Self-Perceived health in functionally independent older people: associated factors. BMC Geriatr 2016;16:66.

39. Leão T, Perelman J. Depression symptoms as mediators of inequalities in self-reported health: the case of southern European elderly. J Public Health 2018;40:756-63.

40. Alarcão V, Madeira T, Peixoto-Plácido C, et al. Gender differences in psychosocial determinants of self-perceived health among Portuguese older adults in nursing homes. Aging Ment Health 2019;23:1049-56.

41. Sonikian M, Metaxaki P, Papavasileiou D, et al. Effects of interleukin- 6 on depression risk in dialysis patients. Am J Nephrol 2010;31:303-8.

42. Nouwen A, Winkley K, Twisk J, et al. Type 2 diabetes mellitus as a risk factor for the onset of depression: a systematic review and metaanalysis. Diabetologia 2010;53:2480-6.

43. Anderson RJ, Freedland KE, Clouse RE, et al. The prevalence of comorbid depression in adults with diabetes: a meta-analysis. Diabetes Care 2001;24:1069-78.

44. Palmer S, Vecchio M, Craig JC, et al. Prevalence of depression in chronic kidney disease: systematic review and meta-analysis of observational studies. Kidney Int 2013;84:179-91.

45. Roy T, Lloyd CE. Epidemiology of depression and diabetes: a systematic review. J Affect Disord 2012;142:S8-21.

46. Matcham F, Rayner L, Steer S, et al. The prevalence of depression in rheumatoid arthritis: a systematic review and meta-analysis. Rheumatology 2013;52:2136-48.

47. Wang S-L, Chang C-H, Hu L-Y, et al. Risk of developing depressive disorders following rheumatoid arthritis: a nationwide populationbased study. PLoS One 2014;9:e107791.

48. Vallerand IA, Patten SB, Barnabe C. Depression and the risk of rheumatoid arthritis. Curr Opin Rheumatol 2019;31:279-84.

49. Lee Y-T, Hu L-Y, Shen C-C, et al. Risk of psychiatric disorders following irritable bowel syndrome: a nationwide population-based cohort study. PLoS One 2015;10:e0133283.

50. Zipfel S, Sammet I, Rapps N, et al. Gastrointestinal disturbances in eating disorders: clinical and neurobiological aspects. Auton Neurosci 2006;129:99-106. 
51. Atlantis E, Baker M. Obesity effects on depression: systematic review of epidemiological studies. Int J Obes 2008;32:881-91.

52. de Wit L, Luppino F, van Straten A, et al. Depression and obesity: a meta-analysis of community-based studies. Psychiatry Res 2010;178:230-5

53. Luppino FS, de Wit LM, Bouvy PF, et al. Overweight, obesity, and depression: a systematic review and meta-analysis of longitudinal studies. Arch Gen Psychiatry 2010;67:220-9.

54. Wang T, Xiao S, Yang C, et al. Prevalence and incidence of depression disorder and cognitive impairment in the elderly in Shanghai, China. Alzheimer's \& Dementia 2015;11:P832-3.

55. Li J, Cacchione PZ, Hodgson N, et al. Afternoon napping and cognition in Chinese older adults: findings from the China health and retirement longitudinal study baseline assessment. J Am Geriatr Soc 2017;65:373-80.

56. Avasthi A, Grover S. Clinical practice guidelines for management of depression in elderly. Indian J Psychiatry 2018;60(Suppl 3):S341-62.

57. Zhang Y, Ting RZ, Yang W, et al. Depression in Chinese patients with type 2 diabetes: associations with hyperglycemia hypoglycemia, and poor treatment adherence. J Diabetes 2015;7:800-8.

58. Fischer MJ, Kimmel PL, Greene T, et al. Elevated depressive affect is associated with adverse cardiovascular outcomes among African Americans with chronic kidney disease. Kidney Int 2011;80:670-8. 